\title{
Use of radiofrequency in cosmetic dermatology: focus on nonablative treatment of acne scars
}

This article was published in the following Dove Press journal:

Clinical, Cosmetic and Investigational Dermatology

12 December 2014

Number of times this article has been viewed

\section{Brian J Simmons \\ Robert D Griffith \\ Leyre A Falto-Aizpurua \\ Keyvan Nouri}

Department of Dermatology and Cutaneous Surgery, University of Miami Miller School of Medicine, University of Miami, Miami, FL, USA

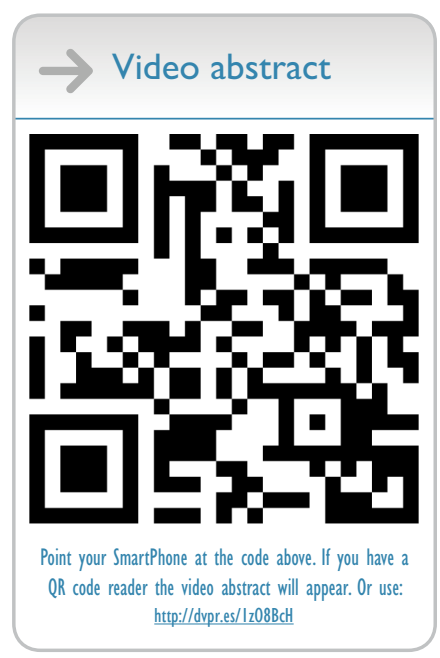

Correspondence: Brian J Simmons University of Miami Miller School of Medicine, Department of Dermatology and Cutaneous Surgery, I475 NW I2th Ave, Miami, FL 33|36, USA

Tel +l 3052433996

Fax +I 3052434184

Email bjsimmons@med.miami.edu

\begin{abstract}
Acne is a common affliction among many teens and some adults that usually resolves over time. However, the severe sequela of acne scarring can lead to long-term psychological and psychiatric problems. There exists a multitude of modalities to treat acne scars such as more invasive surgical techniques, subcision, chemical peels, ablative lasers, fractional lasers, etc. A more recent technique for the treatment of acne scars is nonablative radiofrequency (RF) that works by passing a current through the dermis at a preset depth to produce small thermal wounds in the dermis which, in turn, stimulates dermal remodeling to produce new collagen and soften scar defects. This review article demonstrates that out of all RF modalities, microneedle bipolar $\mathrm{RF}$ and fractional bipolar RF treatments offers the best results for acne scarring. An improvement of $25 \%-75 \%$ can be expected after three to four treatment sessions using one to two passes per session. Treatment results are optimal approximately 3 months after final treatment. Common side effects can include transient pain, erythema, and scabbing. Further studies are needed to determine what RF treatment modalities work best for specific scar subtypes, so that further optimization of RF treatments for acne scars can be determined.
\end{abstract}

Keywords: acne scarring, radiofrequency treatments, nonablative radiofrequency treatments, scars

\section{Introduction}

Acne is an almost ubiquitous disease among teenagers. Between $95 \%$ and $100 \%$ of 16 - to 17 -year-old boys and $83 \%-85 \%$ of 16 - to 17 -year-old girls suffer from acne. ${ }^{1-3}$ Most acne flares settle between the ages of 23 and 25 years old, but can affect up to $1 \%$ of males and $5 \%$ of females who are 40 years old. ${ }^{4}$ The majority of acne affects the face and can lead to scarring in up to $95 \%$ of individuals. ${ }^{5}$ The effects of post-acne scarring can be overwhelming, so much so that it has been indicated as a possible risk factor for suicide. ${ }^{6}$ Any type of acne can have the potential to scar and is a problem in terms of contour and color. ${ }^{7,8}$

\section{The development of acne scars}

Acne scars develop out of the evolution of acne from a noninflammatory process to an inflammatory one that can lead to rupture of the follicular wall. Rupture of the follicular wall leads to release of irritating contents (hair, lipids, keratin, Propionibacterium acnes, etc) into the dermis, leading to a dermal inflammatory reaction that activates the classic and alternative complement pathways. ${ }^{8}$ There is evolution of perifollicular abscesses that usually heal, but if encapsulation is incomplete, this can lead to multichannel fistulous tracks and creation of scars. ${ }^{8}$ 


\section{Types of acne scars}

Acne scars can be broken down into two main categories: atrophic and hypertrophic. Atrophic scars can be further divided into three subtypes: icepick scars, rolling scars, and boxcar scars. Scar classification and subtyping can in turn help guide treatment options. ${ }^{9}$ Icepick scars can extend deep into the dermis which makes them resistant to conventional skin resurfacing options. Rolling scars are wider and have fibrous anchoring in the dermis to the subcutis and, thus, must be treated at a subdermal level. Boxcar scars come in two varieties: shallow or deep. The shallow boxcar scars are more amenable to skin resurfacing treatments while deeper boxcar scars are resistant to these superficial treatments. ${ }^{9}$

\section{Conventional treatments of acne scars}

There are a multitude of treatment options that are traditionally used for the treatment of acne scars. These include many ablative measures such as: chemical peels, subcision, ablative laser, dermabrasion, dermal grafting, punch excision, punch elevation, punch grafting, and surgery. ${ }^{9-11}$ In addition, minimally invasive microneedling with a dermaroller can be used to treat acne scars. ${ }^{12,13}$ Alternatively, reduction of acne scars can be accomplished by using temporary fillers. The problem with many of these procedures is that they are invasive and can lead to an increased risk of depigmentation, scarring, and infections with prolonged healing. ${ }^{14}$

Newer treatment modalities to decrease the propensity for postinflammatory hyperpigmentation and aforementioned problems have emerged such as nonablative fractional lasers and radiofrequency (RF) treatments. Nonablative fractional lasers use an array of laser beams to create microscopic areas of thermal necrosis within the skin. ${ }^{15}$ This induces a rapid healing process within the dermis and upregulation of new collagen production.

$\mathrm{RF}$ treatments are an alternative to conventional methods of acne-scar treatment and can be used as monotherapy or as adjuvant therapy with fractional lasers. Three major types of RF treatments exist: unipolar, bipolar, and fractional. The most basic is the unipolar/monopolar RF device that uses a single electrode and a grounding pad on the skin. This RF modality offers deeper penetration of the dermis but increased pain and discomfort to the patient. ${ }^{16}$ Bipolar RF offers an alternative to unipolar/monopolar RF that can deliver a more focused current to the dermis with less pain due to the need to use lower amounts of energy to achieve the same thermal effect.

Fractional RF uses an array of electrodes that allows for zones of thermal wounds to be made between areas of unaffected zones, thus stimulating dermal remodeling and allowing for a supply of reservoir cells to promote healing. ${ }^{17}$ Variations of fractional RF exist that employ microneedles to deliver electrical current to a particular depth within the dermis that decreases damage to the epidermis. Furthermore, alternative modalities exist such as electro-optical synergy systems that combine RF and lasers. The pretreatment with a nonablative laser lowers the tissue impedance (resistance to flow of current) in the skin to allow deeper penetration of the RFs, decreased level of pain, and reduced amount of RF energy to reach the optimal thermal dose, ${ }^{18}$ thus decreasing collateral effects to the surrounding dermis around the area of scarring and decreasing the propensity for damage, which may include blistering, burns, or inflammatory nodules. ${ }^{19}$

This review will focus on nonablative RF treatments for acne scars to assess the effectiveness of particular methodologies and adverse side effects.

\section{Methods}

A systematic review of the literature was performed on September 3, 2014 to find all articles relevant to the nonablative RF treatments for acne scars. The phrase "radiofrequency AND acne scars" was used to search the National Library of Medicine's PubMed database. This search returned 42 sources, which were subsequently evaluated using their titles and abstracts for relevance. Only articles in English involving human subjects that had full abstracts were included in this review. A total of 18 articles that included clinical trials, case studies, or good background information on RF treatments were used in this review.

\section{Results}

A total of six studies were found on nonablative RF for the treatment of acne scars published between 2003 and 2014. These studies comprised 121 patients that were treated by variations of RF (59 males, 58 females, four unknown). Treatment methods included bipolar RF, unipolar RF, and fractional microneedle RF. Only one of the studies specified specific scar subtyping; the other studies opted for using a severity scale from mild to severe and gave scar location along with other qualitative descriptors. The Fitzpatrick skin types treated ranged from type I to VI with an average number of treatment sessions of three to four. Adverse effects of RF treatment included transient pain, scabbing, ecchymosis, erythema, and dryness. Outcomes to treatment were significant, with the majority of patients in all studies responding to treatment with a reduction in scars of at least $25 \%$ from 1 to 8 months after treatment was complete (see Table 1).

The earliest of the studies by Ruiz-Esparza and $\mathrm{Gomez}^{20}$ included 22 patients with moderate to severe scarring cystic 


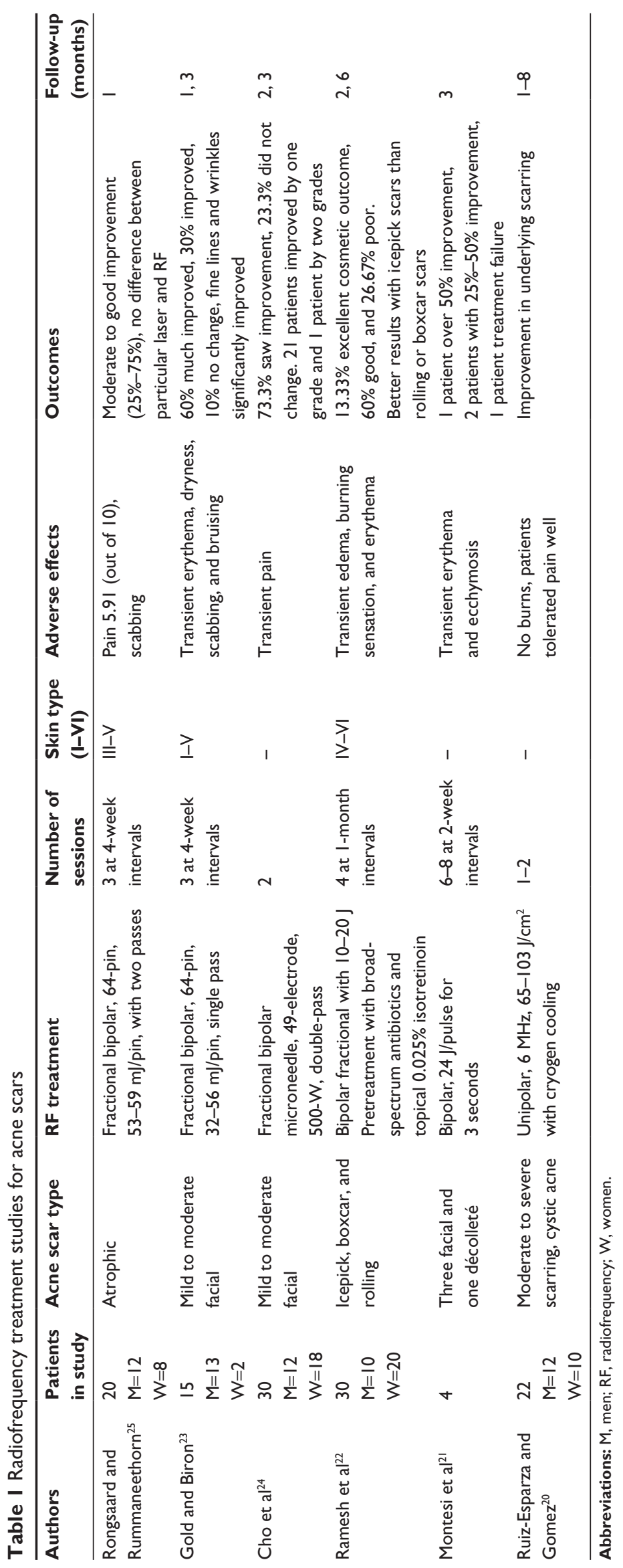


acne. Patients in this study received up to two treatments of unipolar RF with $65-103 \mathrm{~J} / \mathrm{cm}^{2}$ combined with a cooling device. Although the primary endpoint was the resolution of active acne, the underlying scars were noted to improve and results were maintained throughout follow-up of 8 months. In a study by Montesi et al, ${ }^{21}$ four patients with acne scars (three facial and one décolleté) were treated with bipolar RF using $24 \mathrm{~J}$ pulses. Three out of the four responded to treatment with at least $25 \%$ or more improvement in scarring, with no improvement in décolleté scars. Clinical responses became evident after the second treatment session. However, unlike other dermatoses being treated with RF in the study, no histological samples were taken.

Ramesh et $\mathrm{al}^{22}$ utilized a modified approach using a fractionated bipolar RF along with pretreatment with broadspectrum antibiotics and topical isotretinoin. Unlike other studies, which only mentioned treatment of atrophic scars or the severity of the acne scarring, the specific scar type and its response to RF treatment was assessed in this study. Overall, greater than $73 \%$ of patients saw improvement in scarring, similar to findings by Montesi et $\mathrm{al}^{21}$ and Gold and Biron. ${ }^{23}$ However, by looking at specific scar types, it was found that icepick scars responded best to treatment followed by rolling and boxcar, respectively. Of note, eight out of the ten deep boxcar-scar patients underwent subcision prior to RF treatment, most likely due to the deep nature of these boxcar scars.

Gold and Biron ${ }^{23}$ used an alternative RF method employing fractional bipolar RF to treat mild to moderate acne scars. In this study, a single-pass 64-point multi-electrode bipolar RF device was used to deliver three treatment sessions at 4-week intervals. The scars were graded using the ECCA (Echelle d'Evaluation clinique des Cicatrices d'acné) grading system taking into account the significance of scar morphology and age of the scars. The study was able to show "much" improvement in $60 \%$ of patients and improvement in $30 \%$. In addition to improvements in scarring, there were improvements in fine lines, wrinkles, skin tightness, and pigmentation. Patient satisfaction was high and tolerance of the treatment regimens was handled well with no erythema or pigmentation problems at 3 months' follow-up. Of note, additional side effects not seen in other studies (bruising and transient erosions) were seen.

Cho et $\mathrm{al}^{24}$ treated a similar acne scar type to Gold and Biron $^{23}$ (mild to moderate). However, the RF treatment modality was different. A bipolar fractional microneedle RF device using a double-pass method was employed. This specific modality can deliver RF treatment to a deeper depth than more traditional modalities by puncturing the skin with microneedle electrodes. Twenty-two patients (70\%) showed improvement in acne scars after 8 weeks. In addition to improvement in acne scars, skin softening, increased dermal density, and a significant decrease in pore size were noted after 8 weeks. The main problem with fractional RF microneedle in this study was adequate pain control during and several days after treatment, which may be due to the mechanical penetration of the skin with microneedles. Like earlier studies, improvement of acne scars was shown to be due to an increase in production of the dermal matrix, most likely collagen.

Rongsaard and Rummaneethorn ${ }^{25}$ compared bipolar fractional RF to erbium-doped (ED) 1,550 nm laser on 20 patients with Fitzpatrick skin type III-V. A 64-electrode RF device was used with a double-pass technique. RF treatment scored 2.70 out of 5 versus 2.86 for the ED laser treatment when graded by dermatologists, but no statistical difference was seen between the two. Statistically greater improvement in skin texture and shorter periods of scabbing were seen with the ED laser. However, pain scores were significantly higher with ED laser than RF treatments by 1.31 -fold. Thus, both the ED laser and fractional RF treatments significantly improved acne scars, but ED laser treatments were more painful.

\section{Discussion}

Acne scarring can be quite extensive and can cause significant psychosocial and psychological problems for individuals suffering from these post-acne sequelae. A number of modalities have been used to treat acne scars, from invasive surgeries to nonablative RF treatments. Nonablative RF treatments have undergone an evolution from simple unipolar devices to the most recent fractional bipolar microneedle RF modalities. Each subsequent generation of technology has offered more precision to deliver RF energy to deeper tissues with decreased injury to the overlying epidermis, and has allowed for the treatment of all Fitzpatrick skin types (I to VI).

From studies thus far with RF it appears that bipolar fractional microneedle and fractional bipolar RF have shown the best results for resolution of acne scars. A realistic improvement in cosmetic appearance in between $25 \%$ and $75 \%$ of affected skin can be expected with these treatments. It takes an average of three to four treatment sessions with one to two passes and 3 months posttreatment for these results to be fully appreciated. This is most likely due to the required time for adequate activation of fibroblasts and the upregulation of the collagen production needed to replace the dermal matrix. Side effects that can be expected from RF treatments include transient pain, erythema, and scabbing that resolve within 3-5 days; albeit, the pain is significantly less with RF compared to fractional laser treatment. ${ }^{25}$ Besides improvement 
in acne scars, patients may see additional beneficial aesthetic improvements in skin texture, skin tightening, fine lines, and wrinkles, and a reduction in noticeable pore size.

There exists a specific classification system for acne scar type that includes atrophic and hypertrophic scar types, which helps to determine treatment modality, especially in terms of treatment of subtypes of atrophic scars (ie, icepick, boxcar, and rolling). This system has helped to guide conventional treatments of acne scars. However, in all studies except two, the type of acne scarring was not mentioned and only in one study were responses to specific atrophic acne subtypes included. Further investigation that looks at RF treatments with specific outcomes for scar subtype are needed to determine which types of acne scars respond best to RF treatments and if there is variation between specific RF modalities. This will allow for optimization of RF treatments catered to specific acne scar types. Additionally, current studies using RF treatments on acne scarring do not address the long-term sustainability of responses to treatment.

\section{Conclusion}

RF treatments offer an effective alternative for the treatment of acne scars in patients of all skin types, in a small number of treatment sessions, and while offering additional cosmetic benefits. The treatments are minimally invasive and allow for quick recovery times that allow patients to return back to their daily routines the same day. Though the results of this review are promising, more studies with longer follow-up are needed to determine the place of RF in the treatment of acne scarring.

\section{Disclosure}

The authors report no conflicts of interest in this work.

\section{References}

1. Burton JL, Cunliffe WJ, Stafford I, Shuster S. The prevalence of acne vulgaris in adolescence. Br J Dermatol. 1971;85(2):119-126.

2. Munro-Ashman D. Acne Vulgaris in a Public School. Trans St Johns Hosp Dermatol Soc. 1963;49:144-148.

3. Rademaker M, Garioch JJ, Simpson NB. Acne in schoolchildren: no longer a concern for dermatologists. BMJ. 1989;298(6682):1217-1219.

4. Cunliffe WJ, Gould DJ. Prevalence of facial acne vulgaris in late adolescence and in adults. Br Med J. 1979;1(6171):1109-1110.
5. Goodman GJ. Postacne scarring: a review of its pathophysiology and treatment. Dermatol Surg. 2000;26(9):857-871.

6. Cotterill JA, Cunliffe WJ. Suicide in dermatological patients. $\mathrm{Br} J$ Dermatol. 1997;137(2):246-250.

7. Layton AM, Henderson CA, Cunliffe WJ. A clinical evaluation of acne scarring and its incidence. Clin Exp Dermatol. 1994;19(4):303-308.

8. Goodman GJ. Management of post-acne scarring. What are the options for treatment? Am J Clin Dermatol. 2000;1(1):3-17.

9. Jacob CI, Dover JS, Kaminer MS. Acne scarring: a classification system and review of treatment options. J Am Acad Dermatol. 2001;45(1): $109-117$.

10. Tierney EP, Kouba DJ, Hanke CW. Review of fractional photothermolysis: treatment indications and efficacy. Dermatol Surg. 2009;35(10): $1445-1461$.

11. Goodman G. Post acne scarring: a review. $J$ Cosmet Laser Ther. 2003;5(2):77-95.

12. Doddaballapur S. Microneedling with dermaroller. J Cutan Aesthet Surg. 2009;2(2):110-111.

13. Dogra S, Yadav S, Sarangal R. Microneedling for acne scars in Asian skin type: an effective low cost treatment modality. J Cosmet Dermatol. 2014;13(3):180-187.

14. Alexiades-Armenakas MR, Dover JS, Arndt KA. The spectrum of laser skin resurfacing: nonablative, fractional, and ablative laser resurfacing. J Am Acad Dermatol. 2008;58(5):719-737; quiz 738-740.

15. Scheinfeld NS, Goldberg DJ. Nonablative Resurfacing [webpage on the Internet]. Medscape; 2014 [updated February 4, 2014]. Available from: http://emedicine.medscape.com/article/1126583-overview. Accessed September 8, 2014.

16. Lolis MS, Goldberg DJ. Radiofrequency in cosmetic dermatology: a review. Dermatol Surg. 2012;38(11):1765-1776.

17. Hruza G, Taub AF, Collier SL, Mulholland SR. Skin rejuvenation and wrinkle reduction using a fractional radiofrequency system. J Drugs Dermatol. 2009;8(3):259-265.

18. Elsaie ML. Cutaneous remodeling and photorejuvenation using radiofrequency devices. Indian J Dermatol. 2009;54(3):201-205.

19. Sadick NS. Combination radiofrequency and light energies: electro-optical synergy technology in esthetic medicine. Dermatol Surg. 2005;31(9 Pt 2):1211-1217; discussion 1217.

20. Ruiz-Esparza J, Gomez JB. Nonablative radiofrequency for active acne vulgaris: the use of deep dermal heat in the treatment of moderate to severe active acne vulgaris (thermotherapy): a report of 22 patients. Dermatol Surg. 2003;29(4):333-339; discussion 339.

21. Montesi G, Calvieri S, Balzani A, Gold MH. Bipolar radiofrequency in the treatment of dermatologic imperfections: clinicopathological and immunohistochemical aspects. J Drugs Dermatol. 2007;6(9): 890-896.

22. Ramesh M, Gopal M, Kumar S, Talwar A. Novel Technology in the Treatment of Acne Scars: The Matrix-tunable Radiofrequency Technology. $J$ Cutan Aesthet Surg. 2010;3(2):97-101.

23. Gold MH, Biron JA. Treatment of acne scars by fractional bipolar radiofrequency energy. $J$ Cosmet Laser Ther. 2012;14(4):172-178.

24. Cho SI, Chung BY, Choi MG, et al. Evaluation of the clinical efficacy of fractional radiofrequency microneedle treatment in acne scars and large facial pores. Dermatol Surg. 2012;38(7 Pt 1):1017-1024.

25. Rongsaard N, Rummaneethorn P. Comparison of a fractional bipolar radiofrequency device and a fractional erbium-doped glass 1,550-nm device for the treatment of atrophic acne scars: a randomized split-face clinical study. Dermatol Surg. 2014;40(1):14-21.
Clinical, Cosmetic and Investigational Dermatology

\section{Publish your work in this journal}

Clinical, Cosmetic and Investigational Dermatology is an international, peer-reviewed, open access, online journal that focuses on the latest clinical and experimental research in all aspects of skin disease and cosmetic interventions. All areas of dermatology will be covered; contributions will be welcomed from all clinicians and

\section{Dovepress}

basic science researchers globally. This journal is indexed on CAS. The manuscript management system is completely online and includes a very quick and fair peer-review system, which is all easy to use. Visit http://www.dovepress.com/testimonials.php to read real quotes from published authors. 